\title{
Monodisperse Gas-Filled Microparticles from Reactions in Double Emulsions
}

\author{
Wynter J. Duncanson, ${ }^{\dagger}$ Alireza Abbaspourrad, ${ }^{\dagger}$ Ho Cheung Shum, ${ }^{\S}$ Shin-Hyun Kim, ${ }^{\dagger}$ Laura L. A. Adams, ${ }^{\dagger}$ \\ and David A. Weitz ${ }^{*+\dagger} \dagger$
}

${ }^{\dagger}$ School of Engineering and Applied Sciences and ${ }^{\ddagger}$ Department of Physics, Harvard University, Cambridge, Massachusetts 02138, United States

ABSTRACT: We present a strategy for preparing size-controlled gas-filled microparticles using two aqueous components that chemically react to produce the gas. We use a dual-bore microfluidic device to isolate the reactants of two gas-producing reactions until they are encapsulated in the outer droplet. The reactants in the monodisperse droplets merge and produce the gas bubbles, which are stabilized with a surfactant and form the core of the microparticles. The number and size of the generated gas bubbles are governed by the gas-forming reaction used. Our versatile strategy can be applied to a wide range of gas-producing reactions.

\section{INTRODUCTION}

Gas-filled microparticles with controllable sizes and structures are useful as drug-delivery vehicles ${ }^{1-3}$ and ultrasound contrast agents. ${ }^{4}$ Their performance for these applications depends critically on the properties of the microparticles; for example, the microparticle size and gas content determine both the acoustic response ${ }^{5}$ for ultrasound contrast agents and the loading capacity for drug-delivery vehicles. ${ }^{6}$ Thus, the precise control of gas-filled microparticle properties is essential to matching their structure with their function. ${ }^{6,7}$ However, this required control is not possible with conventional synthesis strategies; these rely on the high-shear emulsification of gasnucleating reactants that are photo-, thermally, or chemically initiated. ${ }^{8,9}$ Microparticles produced with these techniques have broad size distributions that limit their utility. These limitations can be overcome by using a microfluidic device to isolate each emulsion phase and generate precisely controlled microparticles. ${ }^{10,11}$ For example, an aqueous gas-forming agent that can be photo- or thermally initiated is encapsulated in monodisperse droplets; ${ }^{12}$ gas evolution inside the droplets is triggered further downstream by heat or UV illumination to yield gas-filled microparticles. However, the direct incorporation of two-component chemically initiated gas-nucleating reactions into microfluidic devices is hindered by disrupted flow patterns and the clogging of the microfluidic channels because of gas-bubble nucleation at undesired locations. Instead, the reactive components must be introduced into the microfluidic device in separate streams. Hence, to use more versatile twocomponent gas-forming agents, a device that enables their separation before encapsulation is required. One such microfluidic device is a glass capillary device with separate compartments for each of the two reactants. ${ }^{13-16}$ This device has been used to produce microfluidic droplets that contain one drop of sodium alginate and another drop of calcium chloride; a hydrogel is formed after the two inner drops coalesce.
However, similar microfluidic devices have not been used to produce gas-filled microparticles with two-component gasforming agents.

In this letter, we use a dual-bore microfluidic device to isolate two gas-forming reactants prior to encapsulating them in monodisperse droplets; the components react to produce gas bubbles within the droplets. The use of microfluidic devices affords precise control of droplet dimensions, and the gasforming agents govern the internal morphology. We also minimize gas dissolution by using colloidal surfactants to stabilize the bubbles.

\section{EXPERIMENTAL SECTION}

Materials. All chemicals were used as received from the manufacturers. Hydrophobic silica nanoparticles (organosilicasol-TolST) were generously provided by Nissan Chemicals America. The shell fluid poly(dimethylsiloxane) (PDMS) oil (Sigma-Aldrich) contained 2 wt \% surfactant (Dow Corning 749) or 40\% Tol-ST. The inner-phase fluid was hydrogen peroxide (30\%) (Sigma-Aldrich) and $0.2 \mu \mathrm{g} / \mathrm{mL}$ catalase (Sigma-Aldrich) or $10 \mathrm{wt} \%$ sodium bicarbonate (Sigma Aldrich) and $0.1 \mathrm{~N}$ hydrochloric acid (SigmaAldrich). The continuous outer phase was composed of $10 \mathrm{wt} \%$ poly(vinyl alcohol) (87-89\% hydrolyzed, average $M_{\mathrm{w}}=13000-23$ 000, Sigma-Aldrich). Ultrapure water with an electrical resistance higher than $18 \mathrm{M} \Omega \mathrm{cm}$ was used in all experiments (Milli-Q Synthesis System, Millipore Corp., Billerica, MA, USA).

Generation of Double Emulsions. Round capillaries with an outer diameter of $1.65 \mathrm{~mm}$ containing two inner channels with diameters of $10 \mu \mathrm{m}$ were used as the injection capillaries, and round capillaries with an outer diameter of $1.5 \mathrm{~mm}$ and an inner diameter of $0.2 \mathrm{~mm}$ were also used (World Precision Instruments, Inc.). These were tapered using a micropipet puller (model P-97 Flaming/Brown puller, Sutter Instrument, Novato, CA, USA). The tapered round

Received: March 2, 2012

Revised: April 1, 2012

Published: April 17, 2012 
(a)
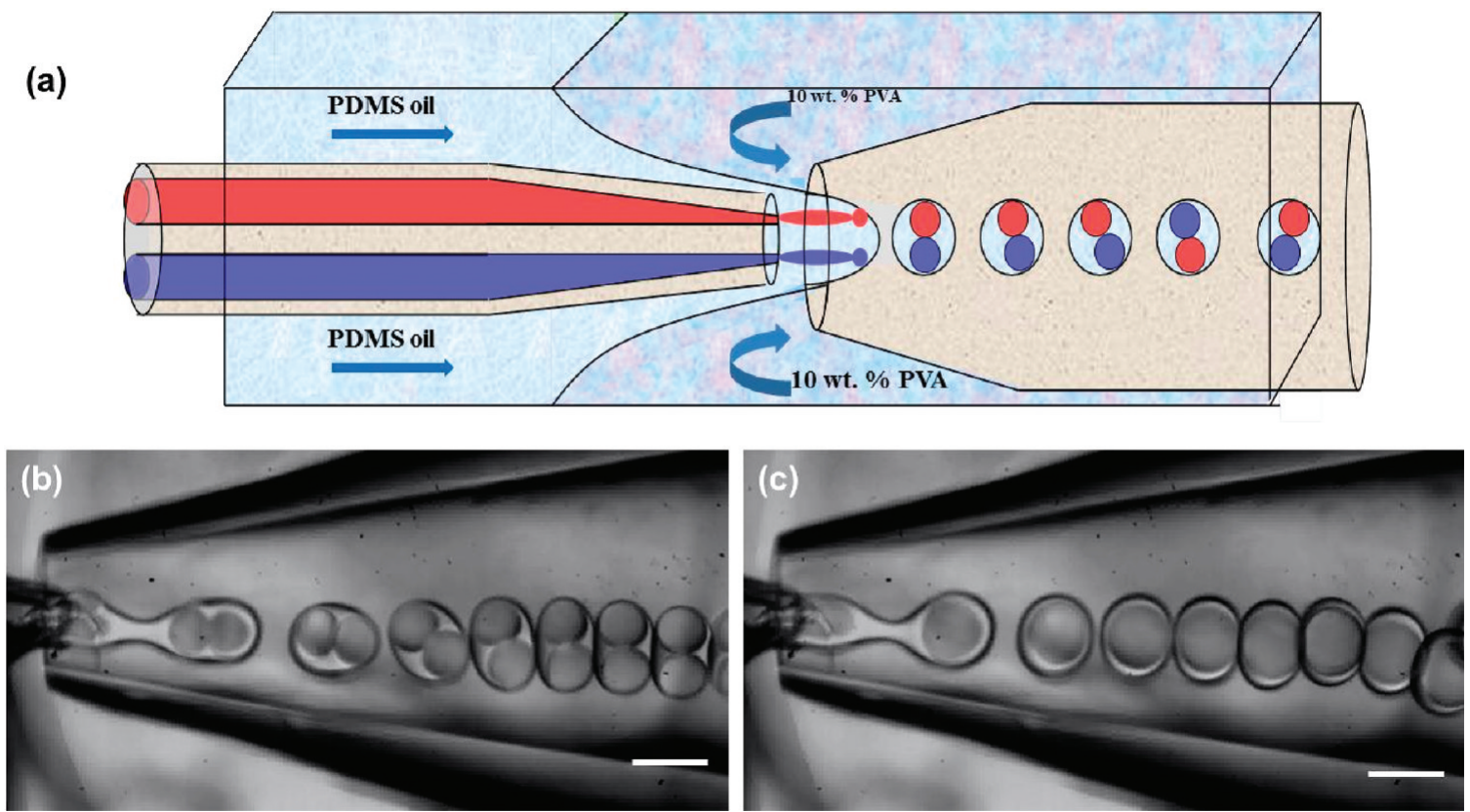

Figure 1. (a) Schematic of the dual-bore microcapillary geometry for generating double emulsions with two distinct inner drops. The injection tube has two separate internal channels, which allow two different fluids to enter the devices separately. (b) The two reactants are encapsulated as two distinct droplets with low inner- and middle-phase flow rates when the innermost channels operate in the dripping regime. (c) The two reactants are encapsulated as a single droplet with high inner- and middle-phase flow rates when the innermost channels operate in the jetting regime. The scale bar is $100 \mu \mathrm{m}$.

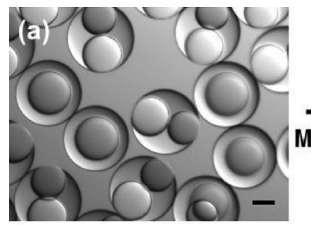

$0 \mathrm{~s}$

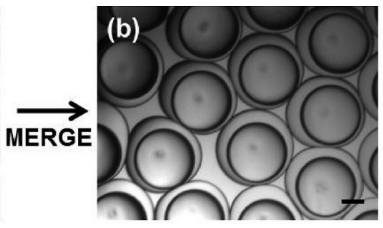

$30 \mathrm{~s}$

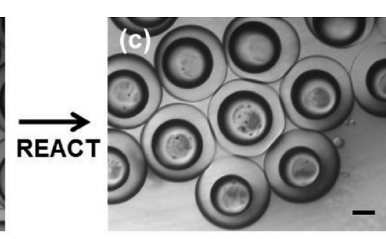

$60 \mathrm{~s}$

Figure 2. Stages of structural evolution of gas in collected droplets. (a) Immediately after collection on glass slides, some drops still contain the two distinct drops whereas others contain coalesced drops. (b) After the coalescence of two initially distinct drops, one inner core forms and gas begins to nucleate within each of the droplets. (c) The nucleation point grows and becomes a gas core within each of the droplets. The scale bar is $50 \mu$ m.

injection and outer capillaries were coaxially aligned with each other in a square capillary with an inner diameter of $1.75 \mathrm{~mm}$ (Atlantic International Technologies). In typical device geometries, an injection taper of $10-30$ (average $=20) \mu \mathrm{m}$ was positioned directly in front of the larger outer collection capillary with a taper of 200-300 (average $=$ 250) $\mu \mathrm{m}$.

Syringe pumps (Harvard Apparatus, PHD2000) were used to regulate the liquid phases in gastight syringes. The syringes were fitted to polyethylene (PE) tubing (Scientific Commodities) and attached to the device positioned on a microscope stage. Typical flow rate ranges for the inner, middle, and outer phases span 500, 1800, and $6500 \mu \mathrm{L} /$ $\mathrm{h}$, respectively.

Sample Characterization. The microfluidic process was monitored using an inverted optical microscope (DM-IRB, Leica) fitted with a fast camera (Phantom V9, Vision Research). Bright-field images were obtained with $10 \times$ objectives at room temperature using an upright microscope (Leica,DMRX) equipped with a digital camera (QImaging, QICAM 12-bit).

\section{RESULTS AND DISCUSSION}

We use glass capillary microfluidic devices to prepare double emulsions that contain two gas-forming aqueous phases. The capillary devices have flow-focusing geometry in which two round-tapered capillaries, one for injection and one for collection, are coaxially aligned within a square capillary, as shown schematically in Figure 1. The injection capillary has two distinct inner channels that separate the reactive components to avoid premature mixing until they are each encapsulated by the coflowing middle phase. The resultant emulsion is subsequently flow-focused by the outer aqueous solution at the orifice of the collection tube, producing monodisperse double emulsions. The inner aqueous phases are hydrogen peroxide and catalase, the middle phase consists of PDMS oil with a surfactant, and the outer phase is a poly(vinyl alcohol) solution.

By adjusting the flow rates, we can control the drop formation of the two innermost fluids to produce a single drop containing the two fluids. Alternatively, we can produce two individual drops, one of each fluid, enabling us to control the onset of the reaction. A single drop is produced when the two inner channels operate in the jetting regime ${ }^{17}$ and the two streams intersect and merge at the orifice, as shown in Figure 1a; gas is produced less than a minute after the two streams mix. By contrast, two drops, each containing one of the innermost fluids, are produced when the two inner channels each operate in the dripping regime, ${ }^{17}$ as shown in Figure $1 \mathrm{~b}$. In both cases, the coflowing middle phase forms the outer droplet that encapsulates the inner drops, as shown in Figure 1; these double emulsion droplets flow downstream and are collected. For the emulsion drops containing two inner droplets, the 

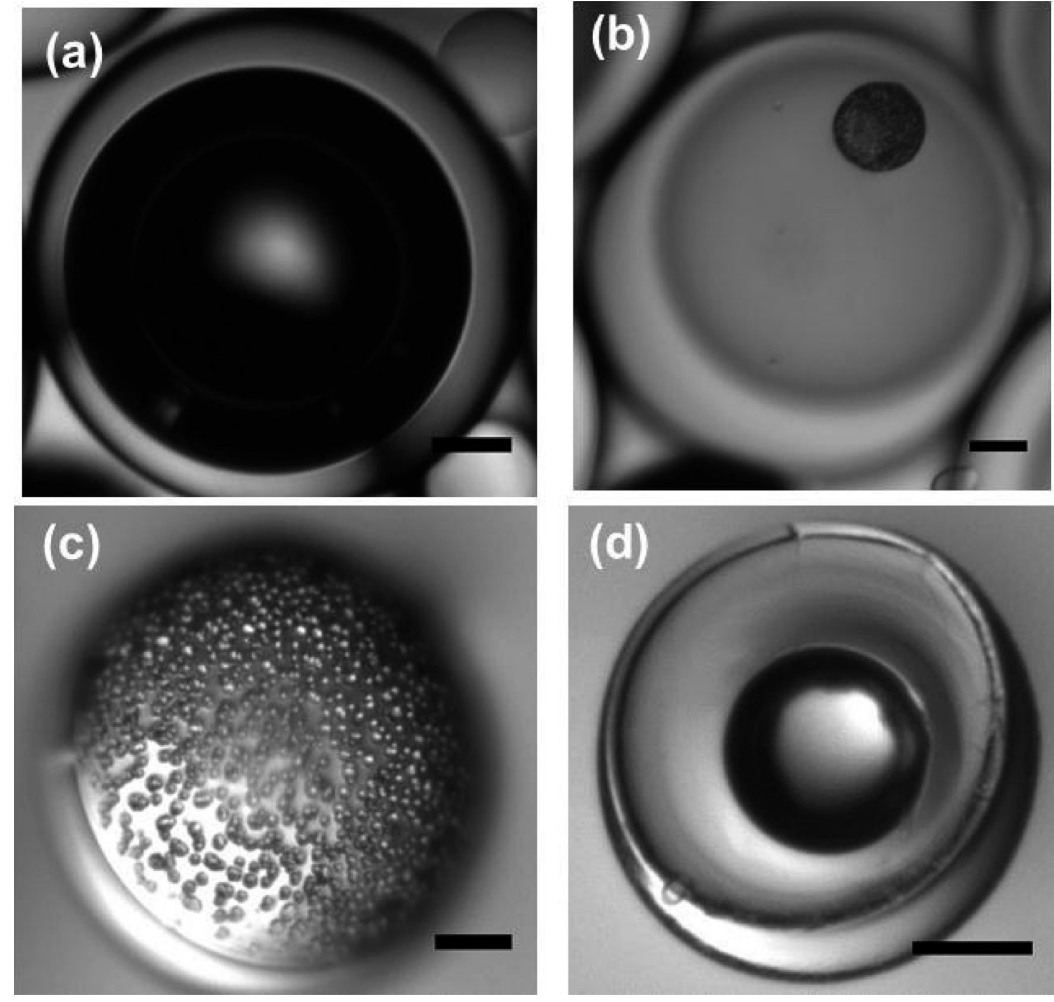

Figure 3. (a) The use of the hydrogen peroxide catalase reaction generates one large gas core of oxygen. (b) After a certain period of time, the gas core retained by the surfactant-stabilized oil shell decreases to $50 \mu \mathrm{m}$. (c) The ammonium bicarbonate reaction generates two gas types that produce many small gas cores. (d) The stability of the oxygen bubble is increased by the addition of a colloidal surfactant in the oil shell. The scale bar is 50 $\mu \mathrm{m}$.

coalescence events are not synchronized; therefore, a fraction of these still contain two unreacted inner droplets, as shown in Figure 2a. These internal droplets move around freely within the larger droplet; this can continue even for a few hours, but ultimately the inner droplets collide and coalesce with one another. ${ }^{18}$ We can monitor these droplets and follow the decomposition of hydrogen peroxide in the presence of catalase as given by

$$
\mathrm{H}_{2} \mathrm{O}_{2}(\mathrm{aq}) \rightarrow \mathrm{H}_{2} \mathrm{O}(\mathrm{aq})+\mathrm{O}_{2}(\mathrm{~g})
$$

The decomposition initially forms one macroscopic dark nucleation point shown in Figure $2 b$; this dark spot confirms the presence of a nucleating gas phase. Within $30 \mathrm{~s}$, the gas phase grows to become a large bubble of oxygen contained within the microparticle, as shown in Figure 2c. After the decomposition is completed, a microparticle containing a single gas core is produced, as shown in Figure 3a.

The degree to which the gas bubble is stabilized within the microparticle is governed by the type of surfactant in the middle phase. When the middle phase contains a conventional surfactant, the encapsulated bubble decreases to a quarter of its initial size over the course of 3 days because of gas dissolution, as shown in Figure $3 \mathrm{~b}$. The use of colloidal surfactants can overcome this limitation because they can effectively stabilize bubbles and foams; ${ }^{19,20}$ therefore, we use nanoparticles as a colloidal surfactant in the preparation of gas-filled microparticles. In this case, after 3 days of storage these bubbles decrease to only $60 \%$ of their initial size, as shown in Figure 3d, whereas the conventional surfactant-stabilized bubble decreases to $25 \%$ of its initial size, as shown in Figure $3 \mathrm{~b}$. This confirms that colloidal surfactants are more effective in preventing gas dissolution in these microparticles.

We can also control the internal microstructure by using an alternate strategy that involves the reaction between sodium bicarbonate and hydrochloric acid. This reaction enables the preparation of droplets that contain two gases, ammonia and carbon dioxide:

$$
\begin{aligned}
& \mathrm{NaHCO}_{3}(\mathrm{aq})+\mathrm{HCl}(\mathrm{aq}) \rightarrow \mathrm{NaCl}(\mathrm{s})+\mathrm{H}_{2} \mathrm{CO}_{3}(\mathrm{aq}) \\
& \mathrm{H}_{2} \mathrm{CO}_{3}(\mathrm{aq}) \rightarrow \mathrm{H}_{2} \mathrm{O}(\mathrm{aq})+\mathrm{CO}_{2}(\mathrm{~g})
\end{aligned}
$$

The intermediate step in the reaction produces solid sodium chloride, which acts as individual nucleation sites ${ }^{21}$ to produce many gas bubbles within the droplets, as shown in Figure 3c. This internal microstructure differs from the single gas that results from the hydrogen peroxide and catalase reaction in eq 1 that is shown in Figure 3a, highlighting our ability to tune the microstructure of the particles through the control of the gasforming reactions.

\section{CONCLUSIONS}

Because gas bubbles are prone to dissolution, even after they have been stabilized, they may rapidly dissolve or coalesce after they have been stored for extended periods ${ }^{22}$ or even shortly after they have been produced. ${ }^{23}$ The strategy introduced in this letter overcomes this limitation because we produce microparticles that contain two aqueous phases that become gaseous only after they coalesce. We can potentially control the timescale of the reaction by controlling the type and amount of surfactant in the microparticle-forming middle phase. Micro- 
particles formed with this strategy are more mechanically robust than traditional prestabilized gas-filled microparticles, thus this strategy has great potential for use in ultrasonic imaging and drug-delivery applications.

\section{AUTHOR INFORMATION}

\section{Corresponding Author}

*E-mail: weitz@seas.harvard.edu.

\section{Present Address}

${ }^{\S}$ Department of Mechanical Engineering, University of Hong Kong, Pokfulam Road, Hong Kong.

\section{Notes}

The authors declare no competing financial interest.

\section{ACKNOWLEDGMENTS}

This work was supported by the Advanced Energy Consortium. Member companies include BP America Inc., Baker Hughes Inc., Conoco-Phillips, Halliburton Energy Services Inc., Marathon Oil Corp., Occidental Oil and Gas, Petrobras, Schlumberger, Shell, and Total.

\section{REFERENCES}

(1) Hauff, P.; Seemann, S.; Reszka, R.; Schultze-Mosgau, M.; Reinhardt, M.; Buzasi, T.; Plath, T.; Rosewicz, S.; Schirner, M. Evaluation of Gas-Filled Microparticles and Sonoporation as Gene Delivery System: Feasibility Study in Rodent Tumor Models. Radiology 2005, 236, 572-578.

(2) Hernot, S.; Klibanov, A. L. Microbubbles in UltrasoundTriggered Drug and Gene Delivery. Adv. Drug Delivery Rev. 2008, 60, 1153-1166.

(3) Seemann, S.; Hauff, P.; Schultze-Mosgau, M.; Lehmann, C.; Reszka, R. Pharmaceutical Evaluation of Gas-Filled Microparticles as Gene Delivery System. Pharm. Res. 2002, 19, 250-257.

(4) Klibanov, A. L. Ligand-Carrying Gas-Filled Microbubbles: Ultrasound Contrast Agents for Targeted Molecular Imaging. Bioconjugate Chem. 2005, 16, 9-17.

(5) Chen, W.-S.; Matula, T. J.; Brayman, A. A.; Crum, L. A. A Comparison of the Fragmentation Thresholds and Inertial Cavitation Doses of Different Ultrasound Contrast Agents. J. Acoust. Soc. Am. 2003, 113, 643-651.

(6) Stride, E.; Edirisinghe, M. Novel Microbubble Preparation Technologies. Soft Matter 2008, 4, 2350-2359.

(7) Feshitan, J. A.; Chen, C. C.; Kwan, J. J.; Borden, M. A. Microbubble Size Isolation by Differential Centrifugation. J. Colloid Interface Sci. 2009, 329, 316-324.

(8) Bae, S. E.; Son, J. S.; Park, K.; Han, D. K. Fabrication of Covered Porous PLGA Microspheres Using Hydrogen Peroxide for Controlled Drug Delivery and Regenerative Medicine. J. Controlled Release 2009, 133, 37-43.

(9) Kim, T. K.; Yoon, J. J.; Lee, D. S.; Park, T. G. Gas Foamed Open Porous Biodegradable Polymeric Microspheres. Biomaterials 2006, 27, $152-159$.

(10) Gunduz, O.; Ahmad, Z.; Stride, E.; Tamerler, C.; Edirisinghe, M. Bioinspired Bubble Design for Particle Generation. J. R. Soc., Interface 2012, 9, 389-395.

(11) Wan, J.; Bick, A.; Sullivan, M.; Stone, H. A. Controllable Microfluidic Production of Microbubbles in Water-in-Oil Emulsions and the Formation of Porous Microparticles. Adv. Mater. 2008, 20, 3314-3318.

(12) Gong, X.; Wen, W.; Sheng, P. Microfluidic Fabrication of Porous Polymer Microspheres: Dual Reactions in Single Droplets. Langmuir 2009, 25, 7072-7077.

(13) Chen, H.; Zhao, Y.; Li, J.; Guo, M.; Wan, J.; Weitz, D. A.; Stone, H. A. Reactions in Double Emulsions by Flow-Controlled Coalescence of Encapsulated Drops. Lab Chip 2011, 11, 2312-2315.
(14) Shum, H. C.; Zhao, Y.-j.; Kim, S.-H.; Weitz, D. A. Multicompartment Polymersomes from Double Emulsions. Angew. Chem., Int. Ed. 2011, 50, 1648-1651.

(15) Sun, B. J.; Shum, H. C.; Holtze, C.; Weitz, D. A. Microfluidic Melt Emulsification for Encapsulation and Release of Actives. ACS Appl. Mater. Interfaces 2010, 2, 3411-3416.

(16) Wang, W.; Xie, R.; Ju, X.J.; Luo, T.; Liu, L.; Weitz, D. A.; Chu, L.-Y. Controllable Microfluidic Production of Multicomponent Multiple Emulsions. Lab Chip 2011, 11, 1587-1592.

(17) Utada, A. S.; Chu, L. Y.; Fernandez-Nieves, A.; Link, D. R.; Holtze, C.; Weitz, D. A. Dripping, Jetting, Drops, and Wetting: The Magic of Microfluidics. MRS Bull. 2007, 32, 702-708.

(18) Gao, F.; Su, Z.-G.; Wang, P.; Ma, G.-H. Double Emulsion Templated Microcapsules with Single Hollow Cavities and ThicknessControllable Shells. Langmuir 2009, 25, 3832-3838.

(19) Binks, B. P. Particles as Surfactants - Similarities and Differences. Curr. Opin. Colloid Interface Sci. 2002, 7, 21-41.

(20) Kim, J.-W.; Lee, D.; Shum, H. C.; Weitz, D. A. Colloid Surfactants for Emulsion Stabilization. Adv. Mater. 2008, 20, 32393243.

(21) Yount, D. E.; Kunkle, T. K. Gas Nucleation in the Vicinity of Solid Hydrophobic Spheres. J. Appl. Phys. 1975, 46, 4484-4486.

(22) Lee, M. H.; Prasad, V.; Lee, D. Microfluidic Fabrication of Stable Nanoparticle-Shelled Bubbles. Langmuir 2010, 26, 2227-2230.

(23) Talu, E.; Lozano, M. M.; Powell, R. L.; Dayton, P. A.; Longo, M. L. Long-Term Stability by Lipid Coating Monodisperse Microbubbles Formed by a Flow-Focusing Device. Langmuir 2006, 22, 9487-9490. 